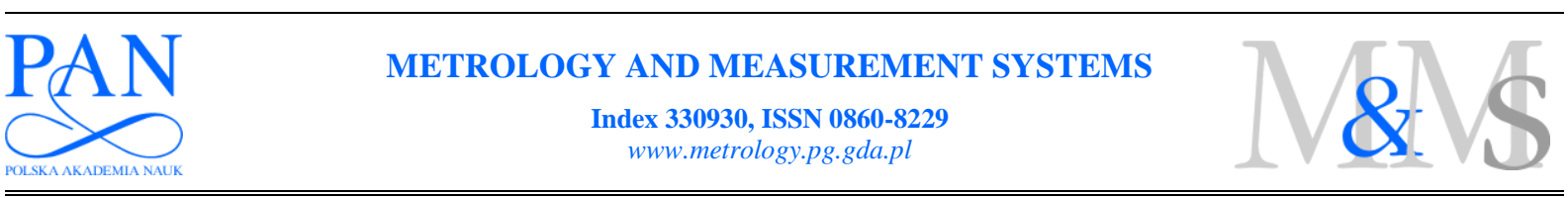

\title{
METROLOGICAL CONDITIONS OF STRAIN MEASUREMENT OPTOELECTRONIC METHOD BY THE USE OF FIBRE BRAGG GRATINGS
}

\section{Piotr Kisala}

Lublin University of Technology, Department of Electronics, 38A Nadbystrzycka Street, 20-618 Lublin, Poland (هp.kisala@pollub.pl)

\begin{abstract}
This article presents a linear strain measurement method insensitive to temperature variations and using fibre Bragg gratings. Two Bragg gratings were applied with periods selected to obtain partial coverage of their spectrum characteristics. One of the gratings was subjected to a tension strength. Placing both gratings in one thermal chamber allowed - through ensuring the same thermal conditions - to obtain insensitivity of the entire scheme to temperature variations. The gratings were recorded on the same optical fibre and reacted to temperature variations in the same degree. Value of strain was indicated based on the transmission spectrum characteristic of two grating schemes. The use of transmission, not reflectance, characteristics of the gratings allowed for a direct measurement of the spectrum, without having to use a coupler or optical circulators, and at the same time, this allowed to simplify the strain detection scheme. We proposed applying the continuous wavelet transform with characteristics of the spectrum scheme of two gratings for improvement of static properties. Especially, the thermal linearity and stability of the sensor was improved. For a strain range up to 750 $\mu \varepsilon$, the non-linearity error of processing characteristic obtained was $0.45 \%$. Thermal stability of the scheme proposed was defined as relative sensitivity of the power to temperature variations. The mean value of such relative sensitivity of the scheme proposed in the temperature scope of $21^{\circ} \mathrm{C}-95^{\circ} \mathrm{C}$, amounted to 0.195 .
\end{abstract}

Keywords: optoelectronic measurement methods, strain measurements, temperature insensitive, fibre Bragg gratings.

(C) 2012 Polish Academy of Sciences. All rights reserved

\section{Introduction}

There are many optoelectronic methods for measuring non-electric quantities. Optical interferometers can measure displacement with a resolution of tens of picometers [1], fibre Bragg gratings (FBG) are commonly used as elements in temperature and strain detection schemes.

In last articles, such as [2], the optical switching and optical bistability phenomenon in fibre grating-based devices have been studied. The nonlinear response of EDF FBG/F-P cavity configuration with two identical fibre Bragg gratings (FBG) separated by an erbiumdoped [3] and $\mathrm{Yb}^{3+}$-doped [4] fibre have been also presented.

Both grating's strain and temperature variations cause a change to Bragg's wavelength. In practice, the problematic issue is the specification of which physical size (temperature or strain) and to what degree caused the Bragg's wavelength shift. Frequently, there is a problem with specifying the elongation or strain value in various environment conditions (for a varying temperature). In many articles the strain measurement using fibre Bragg gratings the temperature is assumed to be constant and the temperature influence on the measurements results is also neglected [5]. Strain distribution recovery using FBG is an indirect measurement example, and it requires inverse problem solution [6,7], also with additionally regularization application [8]. 
There are many solutions allowing to specify the strain and, at the same time, temperature insensitiveness. Such solutions can be divided due to a possibility to use the temperature information into schemes eliminating the temperature impact $[9,10]$, and schemes eliminating the temperature impact, at the same time allowing to measure it (simultaneous strain and temperature measurement) $[11,12]$. Proposed measurement schemes based on FBG can also be divided due to measurement's dynamics into static schemes [13] and dynamic schemes $[14,15]$. A division may be performed as well depending on the temperature compensation method on schemes featuring internal compensation, for example operation [16] where material's features and schemes featuring external compensation are used, for example operation $[17,18]$ where proper installation of gratings on respectively selected mechanical converter is applied.

Measurements methods using fibre Bragg gratings are developed, which are susceptible only to elongation [19] and methods showing variable sensitivity to strain and temperature, but requiring an initial calibration of gratings for every sensitivity, which results in a higher level of complexity of the sole scheme [20].

This paper presents a method that also involves the scheme of two Bragg gratings with relevantly large half-width of spectrum, in order to expand the measurement scope and implementing the wavelet transformation to improve the measurement accuracy and thermal stability. Several time-frequency tools for power signal analysis have been discussed. In general, Gabor Transform, Smoothed Pseudo Wigner-Ville Distribution and Gabor-Wigner Transform analyses are much more computationally complex than Discrete Dyadic Wavelet Transform [21]. In general, the wavelet transform has been used for signal decomposition [22], signal compression, acoustic analysis in the music industry [23]. In this study wavelet transform has been used to denoise signals.

Moreover, strain value is specified based on the transmission spectrum of the entire scheme, which eliminated the need to apply an optical coupler causing reduction of the light power level, as it takes place in case of measurements of reflective spectra.

\section{Measurement principle}

Enforcement to the FBG1 tensed grating is directed through an adhesive link which bonds the tested structure (measurement sample), subject to tension, with the Braggs grating located on the single mode fibre.

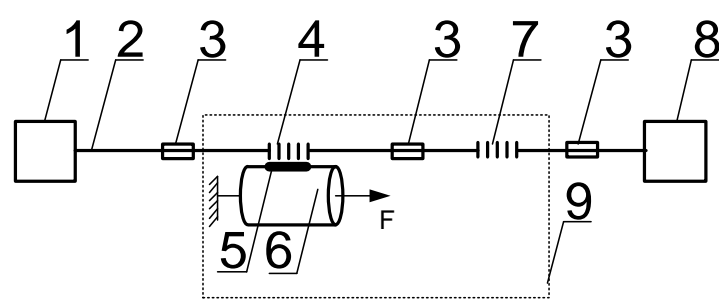

Fig. 1. Block diagram for strain measurements, insensitive to temperature: 1 - white light source, 2 - single mode optical fibre with recorded Bragg grating, 3 - optical fibre link, 4- measurement Bragg grating, 5 - adhesivebonded link, 6 - element examined (sample strained with force F), 7 - reference Bragg grating, 8 - optical spectrum analyzer, 9 - thermal chamber.

The grating is illuminated with a broadband light stream from a white light source. The spectrum after passing through the FBG1 grating is sent to the FBG2 grating. Both gratings are placed in a thermal chamber (Fig. 2) with a regulated and measured temperature, but only the FBG1 grating is adhered to the measurement sample being tensed due to sample's 
elongation. Yet, both gratings are placed at the same experiment-related time-variable temperature. A photo of the measurement post with key elements has been placed in Fig. 3 .
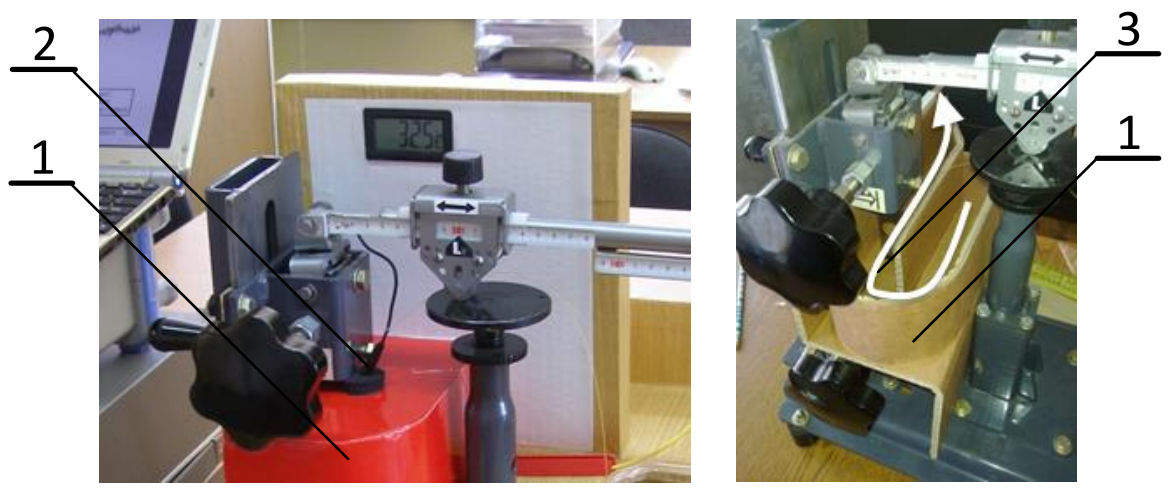

Fig. 2. Thermal chamber providing strain measurement in various temperature conditions: 1 - chamber enclosure, 2 - thermometer probe, 3 - flow direction of input air, with a regulated temperature.

The total of transmission spectra - shifted due to FBG1 gratings strain and, enforcement applied, independent FBG2 grating spectrum - is calculated using the optical spectrum analyzer.

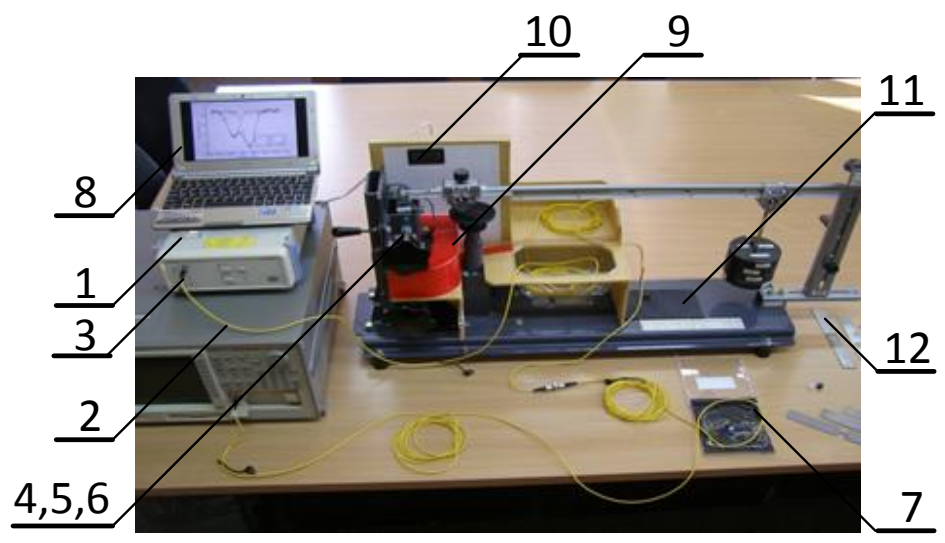

Fig. 3. Laboratory post for strain measurements for a specific temperature: 1 - white light source, 2 - single mode optical fibre with recorded Bragg grating, 3 - optical fibre link, 4 - measurement Bragg grating, 5 - adhesive-bonded link, 6 - element examined (sample strained with force), 7 - reference Bragg grating, 8 - optical spectrum analyzer, 9 - thermal chamber, 10 - thermometer, 11 - laboratory post to generate mechanic loads, 12 - samples tested (sheared).

Both the gratings are recorded on the same fibre, therefore we assume that they have the same thermal expansion coefficient. Moreover, the gratings are placed in the same temperature conditions in order to make the measurement independent from the temperature.

Strain is the power function transmitted through the scheme of two Bragg gratings. Fig. 4 contains measurement outcomes of transmission spectra of specific gratings individually, as well as intended spectrum of two gratings scheme (FBG1 and FBG2). Spectrum characteristics were measured using the optical spectrum analyzer (OSA). Schemes in Fig. 4 have already been averaged using OSA and, nevertheless, prove a significant content of characteristic high-frequency elements which substantially impact the measurement error of the scheme's power, and hence the error in indicating the strain value. The proposed method of indicating the stress layout through simple processing measurement signals from OSA allows to mitigate the above mentioned errors. 


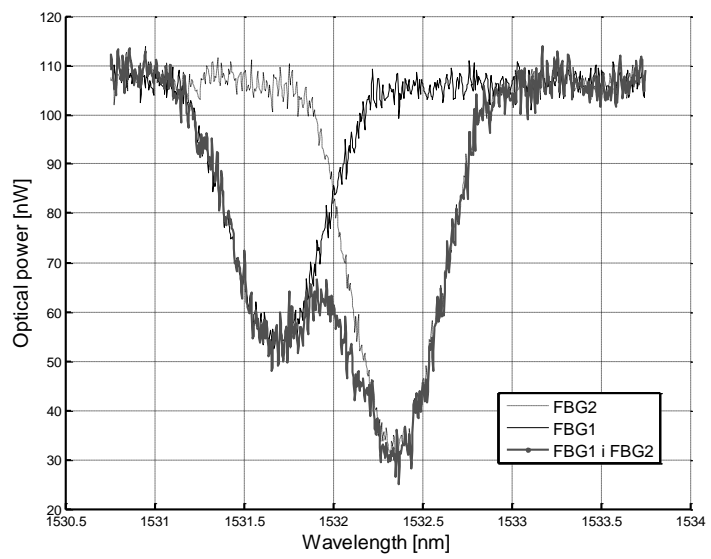

Fig. 4. Transmission spectra measured: FBG2 - dotted black line, FBG1 - continuous black line, FBG1 and FBG2 - continuous grey line. Measurements carried out at the temperature of $20^{\circ} \mathrm{C}$ for gratings strain values $0 \mu \varepsilon$ (non-strained gratings).

Application of the shear force on the grating causes its elongation, i.e. change of its period. Such a change to the period causes a shift of the Bragg's wavelength (and the entire spectrum) towards longer waves, according to the following relation [24]:

$$
\lambda_{B}=2 \pi n_{e f f} \Lambda,
$$

where $\lambda_{B}$ is the middle wavelength of the input light which will be reflected backward from the fibre Bragg grating (Bragg's wavelength), $n_{\text {eff }}$ is an effective refraction coefficient of the fibre core's for the middle wavelength and $\Lambda$ is the grating's period.

If we stretch only FBG1, the FBG1 Bragg's wavelength will be shifted towards longer waves, which will cause a stronger overlapping of the spectra (Fig. 5). As a result, this will reduce the power of FBG1 and FBG2 scheme due to reducing its transmission coefficient. The transmission power of the entire scheme depends therefore on the FBG1 grating strain value. Fig. 5 shows the variation of the power transmitted through the FBG1 and FBG2 schemes in case of strain the FBG1 scheme by $300 \mu \varepsilon$.

The tests use uniform gratings and because the chirp does not occur in uniform gratings (zero value), the formula of the total transmission coefficient can be simplified as follows [25]:

$$
T(z)=\frac{\gamma_{B} \cosh \left[\gamma_{B}\left(z-\frac{L}{2}\right)\right]-i \hat{\sigma} \sinh \left[\gamma_{B}\left(z-\frac{L}{2}\right)\right]}{i \hat{\sigma} \sinh \left(\gamma_{B} L\right)+\gamma_{B} \cosh \left(\gamma_{B} L\right)},
$$

where $L$ is grating's length, $z$ is the grating's examination position, and $\hat{\sigma}$ is a self-coupling coefficient and for the uniform grating equals $\hat{\sigma}=\delta+\sigma$, where $\delta$ is a detuning parameter, and $\sigma$ is equal to [25]:

$$
\sigma=\frac{2 \pi}{\lambda} \overline{\delta n_{e f f}}
$$

where $\overline{\delta n_{e f f}}$ is the background refractive index change, whereas $\gamma_{B}$ is expressed by the following relation [24]: 


$$
\begin{gathered}
\gamma_{B}=\sqrt{\kappa^{2}-\hat{\wedge}^{2}} \text { for } \kappa^{2}>\hat{\sigma}^{2}, \\
\gamma_{B}=i \sqrt{\frac{\wedge^{2}}{\sigma-\kappa^{2}}} \text { for } \kappa^{2}<\hat{\sigma}^{2} .
\end{gathered}
$$

Therefore, the transmission spectrum, after acknowledging the detuning dependency from the wavelength, is as follows

$$
T(z)=\frac{\gamma_{B}^{2}}{\left[2 \pi n_{e f f}\left(\frac{1}{\lambda}-\frac{1}{\lambda_{B}}\right)+\frac{2 \pi}{\lambda} \overline{\delta n_{e f f}}\right]^{2} \sinh ^{2}\left(\gamma_{B} L\right)+\gamma_{B} \cosh ^{2}\left(\gamma_{B} L\right)} .
$$

The spectra obtained from the measurements with the optical spectrum analyzer contained many high-frequency components. In order to limit them and improve the accuracy of measurements, the transmission spectrum was subjected to continuous wavelet transform processes. The new scale and shift parameter function shall read as follows:

$$
S_{\Psi}(a, b)=\frac{1}{\sqrt{a}} \int_{-\infty}^{+\infty} \frac{\gamma_{B}{ }^{2}}{\left[2 \pi n_{\text {eff }}\left(\frac{1}{\lambda}-\frac{1}{\lambda_{B}}\right)+\frac{2 \pi}{\lambda} \overline{\delta n_{\text {eff }}}\right]^{2} \sinh ^{2}\left(\gamma_{B} L\right)+\gamma_{B} \cosh ^{2}\left(\gamma_{B} L\right)} \Psi\left(\frac{\lambda-b}{a}\right) d \lambda,
$$

where $a$ is the scale's parameter, $b$ is the shift's parameter, $\Psi$ is the wavelet function, $S_{\Psi}(a, b)$ is the wavelet coefficient, dependent from the parameters of $a$ and $b, \Psi\left(\frac{\lambda-b}{a}\right)$ is the transformation's core. For all transmission spectra cases, the Daubechies 5 level analyzing function was applied as the wavelet function [27].

Fig. 5 presents the impact of continuous wavelength transform application on the shape of the measured spectrum. Therefore, application of continuous wavelength transform to the FBG1 and FBG2 scheme spectra reduced the high-frequency components from the characteristic.

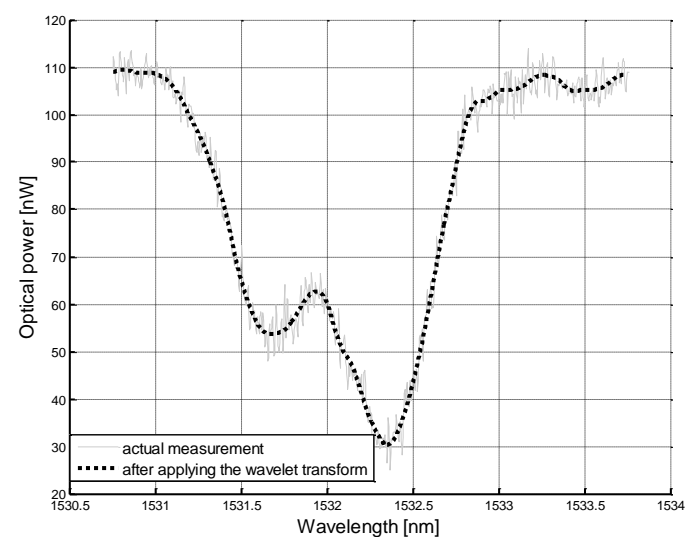

Fig. 5. Spectra characteristics of the FBG1 and FBG2 scheme: actual, obtained from measurements (grey continuous line) and modified through application of the continuous wavelength transform (black dotted line).

Fig. 6 also presents the spectra characteristic of the FBG1 and FBG2 schemes, before and after applying continuous wavelength processing. Fig. 6 contains the spectrum fragment in the 
area of the wavelength where the minimum power occurs ("Bragg's wavelength of the FBG1 and FBG2 scheme").

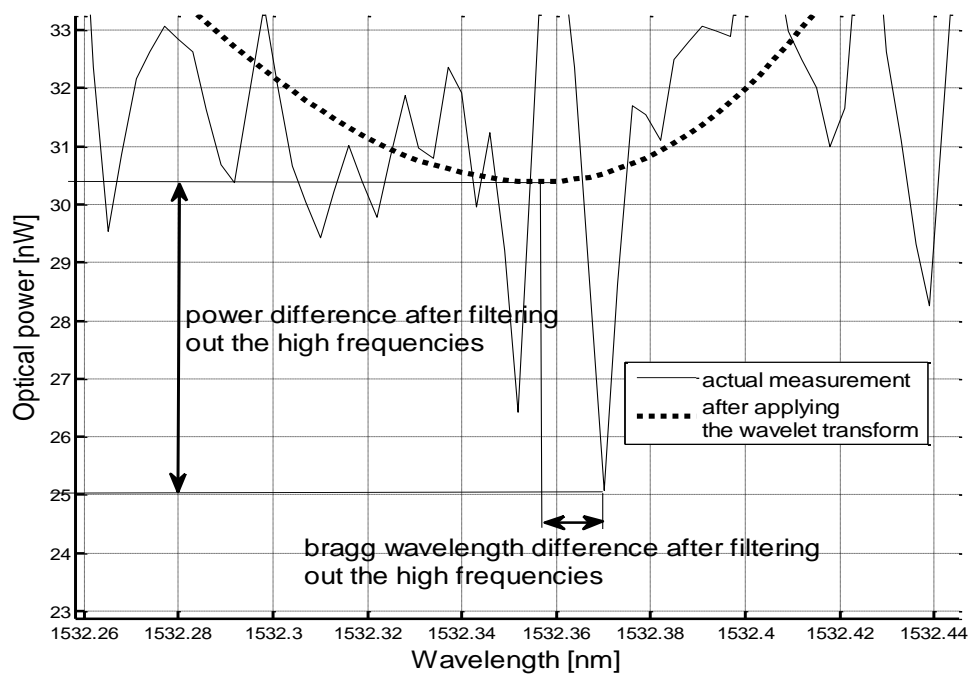

Fig. 6. Spectra characteristics of the FBG1 and FBG2 scheme next to the wavelength for the minimum value of the optical scheme power. Arrows were used to specify the differences between the optical power and wavelength, where the minimum power occurs for both characteristics before and after applying the continuous wavelength transform.

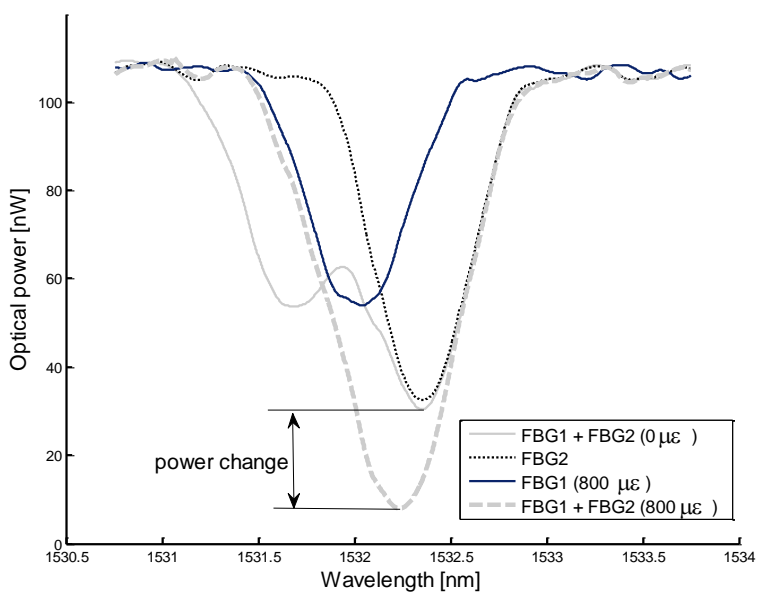

Fig. 7. Spectra characteristics: FBG1 and FBG2 schemes in case of absence of strain (grey continuous line), FBG2 gratings (black dotted line), FBG1 gratings subjected to $800 \mu \varepsilon$ strain and FBG1 and FBG2 scheme in case of FBG1 subjected to $800 \mu \varepsilon$ strain.

Therefore, the application of the wavelet transform caused the wavelength - with the minimum scheme spectrum - to change (Fig. 6). Fig. 7 presents the FBG1 and FBG2 spectra schemes after the application of wavelet transform for the non-strained $(0 \mu \varepsilon)$ and strained $(800 \mu \varepsilon)$ FBG1 grating. The figure presents the reaction of the scheme for FBG1 strain, in the form of the minimum power level change.

\section{Results of measurements and calculations}

Relations-related outcomes of the optical power of the gratings' scheme, before applying the wavelet transform, has been presented in Fig. 8. The figure depicts sizes with the use of 
which the non-linearity error was indicated - equation (7). It may be noted that the linear scope of power to strain dependency amounts to $800 \mu \varepsilon$. The non-linearity error value was fixed from the following relation [28]:

$$
\delta_{n l}=\frac{\Delta P_{M A X}}{P_{M A X}-P_{M I N}} \cdot 100 \%,
$$

where: $\Delta P_{M A X}$ - the maximum value of absolute values of differences between the straightline estimated based on the equation $p_{\text {reg }}(\varepsilon)$ and measurement results $p=f(\varepsilon), P_{M A X}-$ maximum value of $p=f(\varepsilon), P_{M I N}-$ minimum value of $p=f(\varepsilon)$.

The non-linearity error in case of not applying the wavelet transformation amounted to $\delta_{n l}=8.36 \%$.

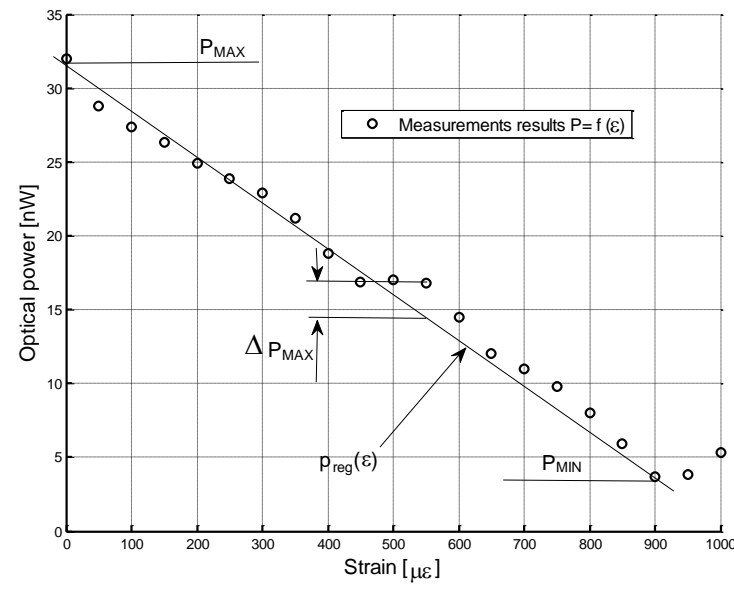

Fig. 8. Chart presenting correlations between the optical power $\mathrm{P}$ in the grating strain function prior to applying the wavelet transformation of measured gratings with a spectrum characteristic.

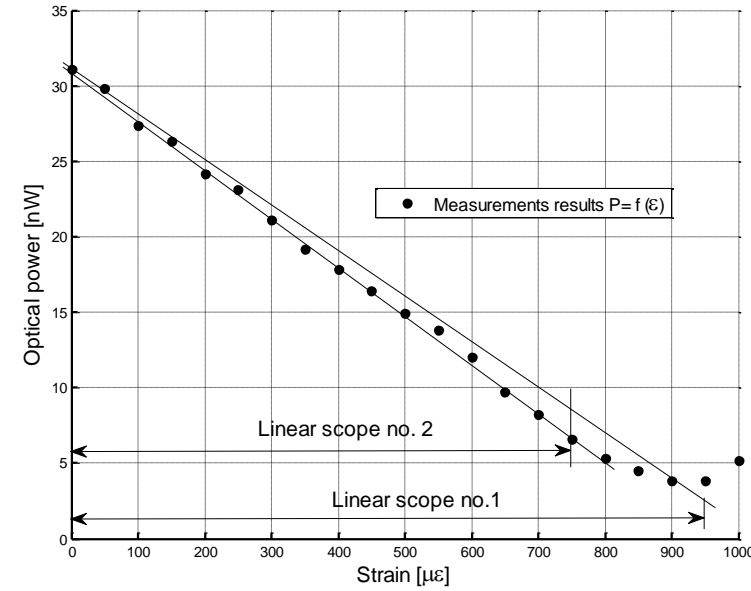

Fig. 9. Chart presenting correlations between the optical power $\mathrm{P}$ in the grating strain function after applying the wavelet transformation of measured gratings with a spectrum characteristic.

For comparison purposes, Fig. 9 also presents the scheme of dependency between the optical power of the scheme in the grating strain function (sensor processing characteristic), yet in this case after applying the wavelet transformation. The linear scope obtained in this case (linear scope no. 1 in Fig. 9) amounts to $950 \mu \varepsilon$. The non-linearity error amounts now to $\delta_{n l}=6.2 \%$.

As presented in Fig. 9, in respect of the proposed scheme, after applying the wavelet transform, there is a possibility to indicate the second linearity scope of the processing characteristic (linear scope no. 2 in Fig. 9) which is smaller compared to the linear scope no. 1 $(750 \mu \varepsilon)$, but it features a substantially smaller non-linearity error, equal to $\delta_{n l}=0.45 \%$.

Also, the thermal stability of the proposed sensor measuring strain was tested. The scheme was tested by changing the temperature in the range between $21^{\circ} \mathrm{C}$ and $95^{\circ} \mathrm{C}$, by measuring the optical power. Measurements were made for the FBG1 grating in the non-strained condition and subjected to strain equal to $700 \mu \varepsilon$ (Fig. 10). Application of wavelet transformation plays a large part in case of testing the temperature's stability. In the case without wavelet transform, the temperature stability amounted to $5.85 \%$, and after applying the transform, the temperature stability of the examined scheme amounted to $0.62 \%$ (value of the power measured varies on the level of $\pm 0.62 \%$ for temperatures between $21^{\circ} \mathrm{C}$ and $95^{\circ} \mathrm{C}$ ). 
The ultimate parameter fixed was relative sensitivity $S_{T}^{P}$ (standardized) of the FBG1 and FBG2 schemes' powers to the temperature, which was defined with the following dependency $[29,30]:$

$$
S_{T}^{P}=\frac{\partial P}{\partial T} \frac{T}{P}=\frac{\partial \ln P}{\partial \ln T}
$$

Therefore, it is a product of the mass factor of the variable $T / P$ and the derivative of the partial initial function $\frac{\partial P}{\partial T}$. Such sensitivity specifies the percentage value of the change to the function value, if the said variable increases by $1 \%$ (Fig. 11).

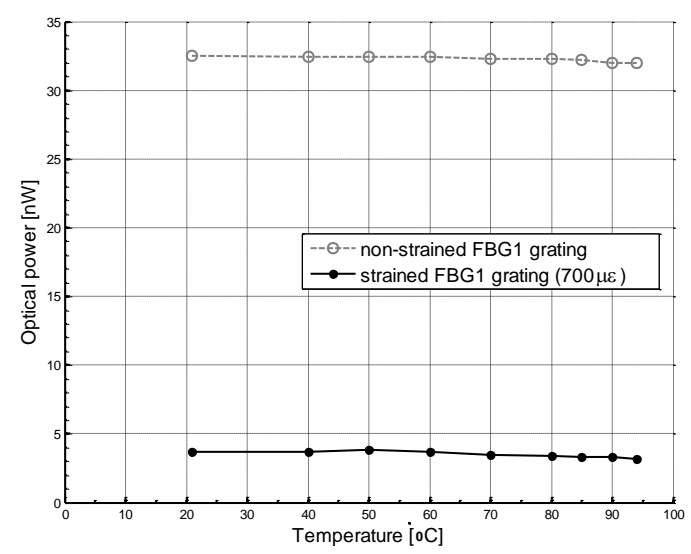

Fig. 10. Chart with correlations of the measured optical power $P$ in the grating temperature function after applying the wavelet transformation in case of the FBG1 non-stained grating (grey dotted line) and subjected to strain with the value of $700 \mu \varepsilon$.

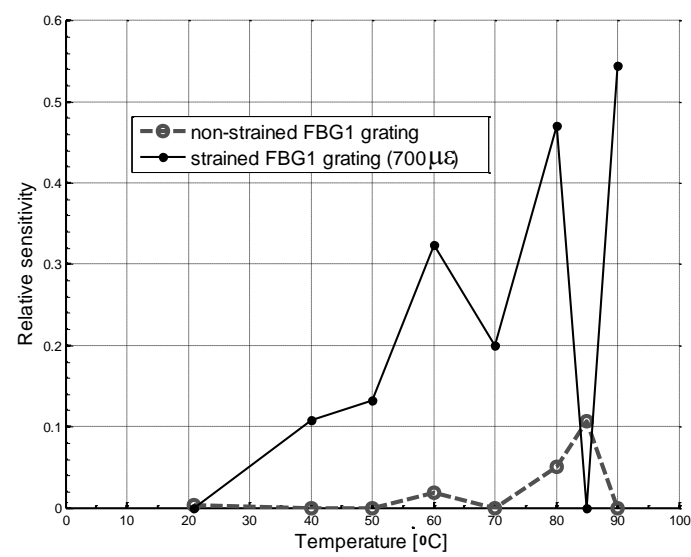

Fig. 11. Chart with correlations of the relative sensitivity in the non-strained FBG1 grating temperature function (grey dotted line) and subjected to strain with the value of $700 \mu \varepsilon$.

The mean relative sensitivity value for the non-strained FBG1 grating amounted to 0.022 , which means that a temperature increase by $1 \%$ will cause a power increase by $0.022 \%$. In the case of grating subjected to strain with the value of $700 \mu \varepsilon$, the mean value of relative sensitivity amounted to $0.195 \%$, which in turn means that a temperature increase by $1 \%$ will cause a power increase by $0.195 \%$. 


\section{Conclusions}

This paper presents an elongation measurement method invulnerable to temperature variations. The elongation was indicated by measuring the minimum power, based on the spectrum characteristic of two Bragg gratings' schemes, which changes along with gratings elongation and temperature variations. By placing two gratings made on the same optical fibre in one thermal chamber, elongation measurement opportunity of one of the gratings was obtained, at the same time maintaining non-sensitivity of this grating to temperature variations. The application of the wavelet transformation of the spectra characteristics of the gratings' schemes obtained allowed to decrease the linearity error of the scheme's processing characteristic from $8.36 \%$ to $0.45 \%$. The proposed method allows to decrease the relative (standardized) sensitivity of the scheme's power to temperature to the level of $0.195 \%$. For an additional decrease of the non-linearity error of the processing characteristic, correction algorithms should be applied.

\section{References}

[1] Wierzba, P. (2008). Stability of an optical displacement sensor using a two-beam polarization interferometer. Metrol. Meas. Syst., 15(2), 205-213.

[2] Zang, Z.G., Yang, W.X. (2011). Theoretical and experimental investigation of all-optical switching based on cascaded LPFGs separated by an erbium-doped fiber. Journal of applied physics, 109, 103106.

[3] Zang, Z.G. (2012). Numerical analysis of optical bistability based on Fiber Bragg Grating cavity containing a high nonlinearity doped-fiber. Optics Communications, 285, 521-526.

[4] Zang, Z.G., Zhang, Y.J. (2012). Low-switching power ( $<45 \mathrm{~mW})$ optical bistability based on optical nonlinearity of ytterbium-doped fiber with a fiber Bragg grating pair. Journal of Modern Optics, 59(2), 161-165.

[5] Wójcik, W., Kisała P. (2009). The application of inverse analysis in strain distribution recovery using the fibre Bragg grating sensors. Metrol. Meas. Syst., 16(4), 649-660.

[6] Mroczka, J., Szczuczyński, D. (2009). Inverse problems formulated in term of first-kind fredholm integral equations in indirect measurements. Metrol. Meas. Syst., 16(3), 333-357.

[7] Mroczka, J., Szczuczyński, D. (2010). Improved regularized solution of the inverse problem in turbidimetric measurements. Applied Optics, 49(24), 4591-4603.

[8] Mroczka, J., Szczuczyński, D. (2012). Summary results of simulation research on improved regularized solution of inverse problem in spectral extinction measurements. Applied Optics, 51(10).

[9] Du, W.C., Tao, X.M., Tam, H.Y. (1999). Temperature independent strain measurement with a fiber grating tapered cavity sensor. IEEE Photon Technol. Lett., 11(5), 596-598.

[10] Guan, B.O., Tam, H.Y., Chan, H.L.W., Choy, C.L., Demokan, M.S. (2002). Discrimination between strain and temperature with a single fiber Bragg grating. Microw. Opt. Technol. Lett., 33(3-5), 200-202.

[11] Da-Peng, Zhou, Li, W., Wing-Ki, L., John, W.Y.L. (2008). Simultaneous measurement of strain and temperature based on a fiber Bragg grating combined with a high-birefringence fiber loop mirror. Optics Communications, 281, 4640-4643.

[12] Fang, Xie, Xianfeng, Chen, Lin, Zhang, Ming, Song. (2006). Realisation of an effective dual-parameter sensor employing a single fibre Bragg grating structure. Optics and Lasers in Engineering, 44, 1088-1095.

[13] James, S.W., Dockney, M.L., Tatam, R.P. (1996). Simultaneous independent temperature and strain measurement using in-fibre Bragg grating sensors. Electron. Lett., 32(12), 1133-1134.

[14] Chan, T.H.T., Yu, L., Tam, H.Y., Ni, Y.Q., Liu, S.Y., Chung, W.H., Cheng, L.K. (2006). Fiber Bragg grating sensors for structural health monitoring of Tsing Ma bridge: Background and experimental observation. Engineering Structures, 28, 648-659. 
[15] Moyo, P., Brownjohn, J.M.W., Suresh, R., Tjin, S.C. (2005). Development of fiber Bragg grating sensors for monitoring civil infrastructure. Engineering Structures, 27, 1828-1834.

[16] Fernández-Valdivielso, C., Matías, I.R., Arregui, F.J. (2002). Simultaneous measurement of strain and temperature using a fiber Bragg grating and a thermochromic material. Sensors and Actuators A, 101, 107-116.

[17] Sungchul, K., Jaejoong, K., Sungwoo, K., Byoungho, L. (2000). Temperature-independent strain sensor using a chirped grating partially embedded in a glass tube. IEEE Photon. Technol. Lett., 12(6), 678-680.

[18] Xu, M.G., Dong, L., Reekie, L., Tucknott, J.A., Cruz, J.L. (1995). Temperature-independent strain sensor using a chirped Bragg grating in a tapered optical fibre. Electron. Lett., 31(10), 823-825.

[19] Kang, S.C., Kim, S.Y., Lee, S.B., Kwon, W., Choi, S.S., Lee, B. (1998). Temperature-independent strain sensor system using a tilted fiber Bragg grating demodulator. IEEE Photon. Technol. Lett. 10, 1461-1463.

[20] Guan, B.O., Tam, H.Y., Tao, X.M., Dong, X.Y. (2000). Simultaneous strain and temperature measurement using a superstructure fiber Bragg grating. IEEE Photon. Technol. Lett., 12, 675-677.

[21] Szmajda, M., Górecki, K., Mroczka, J. (2010). Gabor transform, SPWVD, Gabor-Wigner transform and wavelet transform - tools for power quality monitoring. Metrol. Meas. Syst., 17(3), 383-396.

[22] Adamczak, S., Makieła, W. (2011). Analyzing variations in roundness profile parameters during the wavelet decomposition process using the Matlab environment. Metrol. Meas.Syst., 18(1), 25-34.

[23] Zieliński, T. (2001). Wavelet transform applications in instrumentation and measurement: Tutorial and literature survey. Metrol. Meas. Syst., 5(3), 141-151.

[24] Yu, F.T.S., Yin, S. (2002). Fiber Optic Sensors. Marcel Dekker, 135-136.

[25] Othonos, A., Kalli, K. (1999). Fiber Bragg Gratings. Fundamentals and Applications in Telecommunications and Sensing. Artech House Optoelectronics Library.

[26] Kashyap, R. (1999). Fiber Bragg Gratings. Academic Press.

[27] Mallat, S. (2009). A Wavelet Tour of Signal Processing. The Sparse Way. Elsevier Inc.

[28] Svilainis, L. (2008). LED PWM dimming linearity investigation. Displays, 29, 243-249.

[29] Sedalishche, V.N. (2006). An estimate of the utilization efficiency of coupled modes of oscillation in piezoresonance measuring instruments. Measurement Techniques, 49(8), 825-829.

[30] Adamczak, S., Bochnia, J., Kundera, C. (2012). Stress and strain measurements in static tensile tests. Metrol. Meas. Syst., 19(3), (in edition). 